\title{
Editorial
}

David R. Bevan

Vancouver, Canada

\section{The cost of}

\section{cancelling surgery}

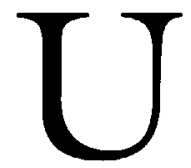

NTIL recently, the emphasis on reducing the cost of anaesthesia and surgery had focused on drugs. ${ }^{1-5}$ Anaesthetists have been urged to revert to older neuromuscular relaxants, ${ }^{2,4}$ modify their use of opioids, ${ }^{3}$ reduce fresh gas flow rate ${ }^{4}$ and be circumspect about incorporating newer, expensive induction agents. ${ }^{1,5}$ Observations that the introduction of propofol at 8-9 times the cost thiopentone resulted in a decrease in costs ${ }^{6}$ make no sense until one realizes the off-setting savings from simultaneously reducing gas flow. Drug costs account for about $10 \%$ of the cost of administering anaesthesia ${ }^{7}$ and, consequently, any savings achieved will be small. We have argued previously that the science of pharmacoeconomics should be concerned with evaluating the total cost of treatment rather than in identifying the cheapest drug available. ${ }^{8}$ Equally, it is difficult not to be cynical when investigators concerned with the development of new drugs also describe their inexpensiveness. ${ }^{9}$ Tuman and Ivankovich reviewed the possible savings resulting from decreasing the use of high technology, such as PA catheters. ${ }^{10}$ Again, overall cost savings are modest as equipment accounts for about $5 \%$ of all anaesthesia costs. ${ }^{?}$

More than three-quarters of the cost of administering anaesthesia is for staff remuneration including that of physicians and nurses. ${ }^{7}$ In addition, Macari $e t$ al. showed that, in Stanford, one third of the hospital costs of the surgical patient are in operating room expenditures and one third are in the surgical ward. ${ }^{11}$ Real savings are much more likely to be achieved by reducing the time patients spend in the operating room, recovery area and surgical wards and thus reducing the staff necessary to care for them. This philosophy has driven the move to ambulatory surgery ${ }^{12}$ and same day admission even for major surgical procedures, ${ }^{13,14}$ and closer examination of $O R$ and PAR efficiency as part of an examination of the costs of the whole surgical experience. ${ }^{15}$

The anaesthetists at the Jewish General Hospital in Montréal have previously analysed the time consequences of spinal or general anaesthesia for gynaeco- logical surgery ${ }^{16}$ (There was no difference.) In the current issue of the Canadian Journal of Anaesthesia they have examined a more difficult but common problem. ${ }^{17}$ How should one proceed at 2 p.m. in an OR planned to finish in $1 \frac{1 / 2}{2}$ hours but with the last case on the list expected to take three hours? Are the costs of continuing (overtime etc) more or less than the costs of cancellation (unused time, etc). The authors have constructed a model of the operational costs of postponing $v s$ continuing with the case and have analysed the process from the perspectives of the payer - patient, society, or hospital. From all perspectives it was more cost-effective to proceed with the case rather than to cancel it. Their conclusion, if valid, may have a considerable influence on decisions late in the afternoon and, as an aside, will give the imaginative surgeon greater room for manipulation. However, such a finding is unlikely to go unchallenged.

The concept that increasing the cost-per-case, by using overtime rates of payment, is cost-effective is intuitively wrong until one realises that cancellation also incurs cost. In the rigid hospital budgetary environment in Canada, hospital staff are paid whether or not work is performed. Cancelling a case will produce unused, but paid, time for OR staff. Efficiency, in terms of the average time to complete cases, will be reduced but hospital costs may not be increased. Indeed, if less work is completed, fee-for-service payments will be reduced. Similarly, reducing the delays between cases will not lead to increased utilisation and greater efficiency unless sufficient time is produced to perform additional cases within the allotted time. Conversely, if additional staff are hired, particularly at overtime rates, the cost to the hospital will increase even if the efficiency improves. Thus, the eventual costs depend, to an extent, on the forms of remuneration.

Others have examined the consequences of modifying the timing of surgical activity with a view to performing the same surgical case load, but with fewer staff. ${ }^{18}$ Dexter and Tinker calculated that if the anticipated duration of surgery and the number of available staff in the PAR were matched, this would lead to con- 
siderable potential savings and the cost benefit would be much greater than the pharmaceutical prevention of postoperative nausea and vomiting. However, it may not be easy to change surgical practice in which the longest and most difficult case is put at the beginning of the list.

The value of these reports is that the approach to value-based medicine is broader than cheap drugs. In the present study, potential patient concerns are also considered. When surgery is cancelled, hospital budgets are preserved at a cost to the patient, who loses time off work, and to those receiving fee-for-service payments. Perhaps it is not surprising that physicians and patients are unanimous in their desire to continue.

\section{Le prix de l'annulation de la chirurgie}

Il n'y a pas de cela si longtemps, on s'attaquait aux prix des médicaments dans le but de couper dans les coûts de l'anesthésie et de la chirurgie. ${ }^{1-5}$ Il était de bonne guerre pour les anesthésistes de revenir aux anciens curarisants, ${ }^{2,4}$ de modifier leur utilisation des morphiniques, ${ }^{3}$ de réduire les débits des gaz frais ${ }^{4}$ et d'y penser deux fois avant d'introduire de nouveaux agents d'induction dispendieux. ${ }^{1,5}$ Implanter le propofol à 8 à 9 fois le coût du thiopental pour réduire les dépenses semblait de la pure folie jusqu'à ce qu'on réalise que la baisse simultanée du débit des gaz procurait des compensations équivalentes sur le plan financier. Le coût des agents anesthésiques ne représentant qu'environ $10 \%$ des coûts de l'anesthésie, ${ }^{7}$ l'économie réalisée ne pouvait donc être que très limitée. Nous avons déjà démontré que la pharmacoéconomie devrait s'intéresser à l'évaluation du coût total d'un traitement plutôt qu'à identifier le moins cher des produits pharmaceutiques sur le marché. ${ }^{8}$ De la même façon, on ne peut s'empêcher de sourire quand on voit les investigateurs responsables de la synthèse de nouveaux produits en vanter la modicité. 9 Truman et Ivankovich se sont attardés à calculer les économies réalisables par la diminution de l'usage des technologies avancées, ex. les cathéters artériels pulmonaires; ${ }^{10}$ encore là, les économies totales ne peuvent être que modestes parce que le matériel ne représente qu'environ $5 \%$ du coût total de l'anesthésie.?

Plus des trois quarts des dépenses occasionnées par l'administration de l'anesthésie proviennent de la rémunération du personnel dont les médecins et le personnel infirmier. ${ }^{7}$ Macari et al. ont aussi montré qu'à Stanford, un tiers des frais encourus par hôpital pour le patient chirurgical provient des dépenses de la salle d'opération et un tiers de celles du service de chirurgie. ${ }^{11}$ Les vraies économies ne peuvent donc résulter que de la réduction de la durée du séjour en salle d'opération, à la salle de réveil et dans le service de chirurgie ainsi que de la décroissance en effectifs du personnel soignant. Cette philosophie a conduit au «virage ambulatoire» ${ }^{12}$ et à la chirurgie d'un jour même pour des interventions chirurgicales majeures ${ }^{13,14}$ et à inciter à la réflexion sur l'efficience de la salle d'opération et de la salle de soins postanesthésiques en tant que facteur modificateur des coûts. ${ }^{15}$

Les anesthésistes de l'Hôpital Général Juif de Montréal ont déjà analysé l'influence de la rachianesthésie comparativement à celle de l'anesthésie générale sur la durée de la chirurgie gynécologique (aucune différence). ${ }^{16}$ Leur étude publiée ce mois-ci dans le Journal canadien d'anesthésie fait état d'un problème fréquent mais plus difficile à résoudre. ${ }^{17}$ Que faire à $2 \mathrm{~h}$ PM dans une salle d'opération devant fermer dans $1 \frac{1 / 2}{h}$, quand la dernière intervention programmée doit durer trois heures ? Les coûts de la prolongation (temps supplémentaire etc.) sont-ils plus élevés ou moins élevés que les coûts de l'annulation (temps inutilisé etc.) ? Les auteurs ont construit un modèle des coûts opérationnels de la chirurgie reportée comparés à ceux de la continuation du programme opératoire et analysé le processus dans la perspective de celui qui paye - le patient, la collectivité ou l'hôpital. Quelle que soit la perspective envisagée, il était plus efficace par rapport au coût d'opérer que d'annuler. Cette conclusion, si elle est valide, peut avoir une influence considérable sur les décisions prises en fin d'après-midi, et, en à-côté, donner plus d'espace pour la manipulation au chirurgien imaginatif. De toutes façons, il est fort probable que la conclusion des auteurs sera contestée.

Le concept selon lequel l'augmentation du coût d'une intervention est évaluée par le paiement de temps supplémentaire défie la raison quand on réalise que l'annulation a aussi ses coûts. Dans la coque rigide des budgets hospitaliers canadiens, le personnel hospitalier est payé, qu'il travaille ou non. Le temps non utilisé à cause de l'annulation sera quand même rémunéré. L'efficience, en terme du temps moyen requis pour compléter une intervention, sera réduite mais les coûts pour l'hôpital n'augmenteront pas nécessairement. En réalité, si on travaille moins, les paiements par la rémunération à l'acte devraient diminuer. De la même façon, la réduction des délais entre les interventions ne 
conduira pas à une augmentation de l'utilisation et à une plus grande efficience à moins que l'espace de temps récupéré permette d'ajouter des interventions à même le temps alloué. Au contraire, s'il faut embaucher du personnel supplémentaire, surtout à des tarifs de temps supplémentaire, il en coûtera plus cher à l'hôpital même si l'efficience s'améliore. Ainsi, les coûts éventuels dépendront, jusqu'à un certain point, du type de rémunération.

D'autres ont étudié les conséquences provoquées par les modifications apportées à l'horaire des activités chirurgicales dans le but d'effectuer le même nombre d'interventions mais avec moins de personnel. ${ }^{18}$ Dexter et Tinker ont calculé que si la durée prévue de la chirurgie était coordonnée avec la disponibilité du personnel de la salle de réveil, il en résulterait des économies considérables et le rapport coût/avantage dépasserait celui de la prévention pharmacologique des nausées et des vomissements postopératoires. Il est toutefois difficile de changer les moeurs chirurgicales qui consistent à inscrire au début du programme les interventions les plus longues et les plus compliquées.

Ces rapports ont le mérite de nous faire réaliser que l'économie va plus loin que l'achat d'agents à vil prix. Dans l'étude actuelle, on tient compte du patient. Quand une intervention est annulée, le budget de l'hôpital est préservé à l'encontre des intérêts du patient qui doit s'absenter du travail et de ceux qui sont rémunérés à l'acte. L'unanimité, entre patients et médecins qui veulent que la journée de travail continue, n'a donc rien de surprenant.

\section{References}

1 Johnstone RE, Martinec CL. Costs of anesthesia. Anesth Analg 1993; 76: 840-8.

2 Orkin FK. Moving toward value-based anesthesia care. J Clin Anesth 1993; 5: 91-8.

3 Hawkes C, Miller D, Martineau R, Hull K, Hopkins H, Tierney $M$. Evaluation of cost minimization strategies of anaesthetic drugs in a tertiary care hospital. Can J Anaesth 1994; 41: 894-901.

4 Becker KE, Carrithers JP. Practical methods of cost containment in anesthesia and surgery. J Clin Anesth 1994; 6: 388-99.

5 Johnstone RE, Lozefczyk KG. Costs of anesthetic drugs: experiences with a cost education trial. Anesth Analg 1994; 78: 766-71.

6 Cooper CG, Maxwell V. Anaesthetic drug costs are not incrcased by propofol (Letter). Can J Anaesth 1992; 39: 1000-4.

7 Astley BA, Secker Walker J. Cost of anaesthetic drugs and clinical budgeting. BMJ 1986; 293: 1587-8.
8 Bevan DR. Anaesthesia pharmacoeconomics. Can J Anaesth 1993; 40: 693-5.

9 Weiskopf RB, Eger EI. Comparing the costs of inhaled anesthetics. Anesthesiology 1993; 79: 1413-8.

10 Tuman KJ, Ivankovich AD. High-cost, high-tech medicine: are we getting our money's worth? J Clin Anesth $1993 ; 5$ : 168-77.

11 Macario A, Vitez TS, Dunn B, McDonald T. Where are the costs in perioperative care? Anesthesiology 1995; 83: 1138-44.

12 Keitbley J, Glandon GL, Llewellyn J, Berger B, Levin D. The cost-effectiveness of same-day admission surgery. Nursing Economics 1989; 7: 90-5.

13 Collier PE. Carotid endarterectomy: a safe cost-efficient approach. J Vasc Surg 1992; 16: 926-33.

14 Hamilton $A$, Norris $C$, Wensel $R$, Koshal $A$. Cost reduction in cardiac surgery. Can J Cardiol 1994; 10: 721-7.

15 Orkin FK. Meaningful cost reduction. Anesthesiology 1995; 83: 1135-7.

16 Tessler MJ, Kardash K, Kleiman S, Rossignol M. A retrospective comparison of spinal and general anesthesia for vaginal hysterectomy: a time analysis. Anesth Analg $1995 ; 81: 694-6$.

17 Tessler MJ, Kleiman SJ, Huberman MM. A "zero tolerance for overtime" increases per case costs. Can J Anaesth 1997; 44: 1036-1041.

18 Dexter $F$, Tinker JH. Analysis of strategies to decrease Postanesthesia Care Unit costs. Anesthesiology 1995; 82: 94-101. 